\title{
Balanço de energia e controle biofísico da evapotranspiração na Caatinga em condições de seca intensa
}

\author{
Luciana Sandra Bastos de Souza ${ }^{(1)}$, Magna Soelma Beserra de Moura(2), Gilberto Chohaku Sediyama(3) \\ e Thieres George Freire da Silva ${ }^{(1)}$
}

\begin{abstract}
(1)Universidade Federal Rural de Pernambuco, Unidade Acadêmica de Serra Talhada, Caixa Postal 063, CEP 56900-000 Serra Talhada, PE, Brasil. E-mail: sanddrabastos@yahoo.com.br, thigeoprofissional@hotmail.com ${ }^{(2)}$ Embrapa Semiárido, BR-428, Km 152, Caixa Postal 23, Zona Rural, CEP 56302-970 Petrolina, PE, Brasil. E-mail: magna.moura@embrapa.br (3)Universidade Federal de Viçosa, Departamento de Engenharia Agrícola, Avenida P.H. Rolfs, s/nº, CEP 36570-000 Viçosa, MG, Brasil. E-mail: g.sediyama@ufv.br
\end{abstract}

Resumo - O objetivo deste trabalho foi determinar a variação sazonal dos componentes do balanço de energia e avaliar o controle biofísico da evapotranspiração, em área de Caatinga preservada, em condições de seca intensa. O experimento foi conduzido em 2012, tendo-se utilizado o sistema "eddy covariance", instalado a $16,9 \mathrm{~m}$ acima da superfície do solo. Além disso, foram realizadas medidas de temperatura, umidade, radiação solar, saldo de radiação, fluxo de calor no solo e temperatura do solo. Os dados dos fluxos de calor sensível e latente foram processados com o programa Alteddy e analisados em escalas diária e sazonal. Com os dados do fluxo de calor latente, foram determinados os valores da evapotranspiração, bem como os indicadores da sua sensibilidade às condições ambientais: fator de desacoplamento, e resistências aerodinâmica e da superfície. O saldo de radiação apresentou forte sazonalidade, com comportamento dependente da radiação solar. A partição dos componentes do balanço de energia revelou que a ocorrência de seca intensa maximiza a destinação da energia disponível ao fluxo de calor sensível, e que a evapotranspiração nessa condição é especialmente dependente do deficit de pressão de vapor e da resistência da superfície.

Termos para indexação: eddy covariance, fator de desacoplamento, fluxo de calor sensível, resistência de superfície, vegetação natural.

\section{Energy balance and evapotranspiration biophysical control in Caatinga under intense drought conditions}

\begin{abstract}
The objective of this work was to determine seasonal variation in energy balance components and to evaluate the biophysical control of evapotranspiration in a preserved Caatinga area, under intense drought conditions. The experiment was conducted in 2012, using the eddy covariance system, installed at $16.9 \mathrm{~m}$ above soil surface. In addition, temperature, humidity, solar radiation, net radiation, soil heat flux, and soil temperature were measured. Data on the sensible and latent heat fluxes were processed with the Alteddy software and analyzed on daily and seasonal scales. Using the latent heat flux data, the evapotranspiration rates were determined, as well as the indicators of their sensitivity to environmental conditions: decoupling factor, and aerodynamical and surface resistances. The net radiation showed a strong seasonality, with behavior dependent on solar radiation. The partition of the energy balance components revealed that the occurrence of intense drought maximizes the destination of the available energy to sensible heat flux, and that evapotranspiration under this condition is specifically dependent on the vapor pressure deficit and on the surface resistance.
\end{abstract}

Index terms: eddy covariance, decoupling factor, sensible heat flux, surface resistance, natural vegetation.

\section{Introdução}

A vegetação de Caatinga recobre a região semiárida brasileira, com extensão territorial de aproximadamente $800.000 \mathrm{~km}^{2}$, compreendendo cerca de $80 \%$ do Nordeste brasileiro e $11 \%$ do território nacional (Santos et al., 2012). O clima local é sazonal, com meses chuvosos, de janeiro a abril, e longo período sem chuvas, de maio a dezembro (Moura et al., 2007). Nos meses secos, a demanda evaporativa da atmosfera torna-se substancialmente elevada e provoca estresse hídrico nas espécies vegetais, o que pode ser potencialmente agravado pelas mudanças nos padrões atmosféricos de larga escala. Essas mudanças foram observadas em 2012 com a ocorrência do fenômeno do Dipolo do Atlântico, que culminou em alterações no regime pluvial da região e, consequentemente, na intensidade da seca (Centro..., 2014; Gutiérrez et al., 2014). 
Uma das formas de avaliar as respostas dos ecossistemas às condições ambientais é por meio do balanço de energia, representado pelas suas partições nos fluxos de calor latente, de calor sensível e de calor no solo (Krishnan et al., 2012; Chen et al., 2009). Há diversos estudos sobre os fluxos de energia e de massa em diferentes superfícies vegetadas (Huizhi et al., 2012; Hu et al., 2009), como, também, sobre a dependência destes em relação às variáveis biofísicas e meteorológicas (Chen et al., 2009; Yang et al., 2011). Em regiões semiáridas, a maior parte da energia disponível é utilizada no aquecimento do ar e do solo, em razão da baixa disponibilidade hídrica (Chen et al., 2009; Teixeira et al., 2008; Oliveira et al., 2006). Para a Caatinga, estudos em campo têm mostrado que, em anos em que a precipitação se aproxima da normal climatológica, essa partição é da ordem de 63, 24,7 e $0,2 \%$ para fluxos de calor sensível, de calor latente e de calor no solo, respectivamente (Teixeira et al., 2008). No entanto, essa partição pode ser alterada de acordo com as variações no regime de precipitação (Teixeira et al., 2008).

Recentemente, vários modelos têm sido aplicados para o estudo do balanço de energia em áreas de Caatinga, e os dados obtidos são utilizados para analisar possíveis alterações nas trocas entre a superfície e a atmosfera, resultantes da mudança do uso da terra (Paiva \& Cavalcanti et al., 2011; Cunha et al., 2013). Porém, pouco se sabe sobre a magnitude e o comportamento desses fluxos em condições de seca intensa. Entre os componentes do balanço de energia, a transferência do vapor d'água tem apresentado maior destaque em análises hidrológicas e agrometeorológicas, e pode ser mais bem compreendida por meio das resistências aerodinâmica e da superfície, bem como do fator de desacoplamento (Silva et al., 2012; Marin \& Angelocci, 2011). Este indicador foi proposto por McNaughton \& Jarvis (1983), e seus valores podem oscilar entre zero e um, os quais representam o controle da evapotranspiração pela vegetação ou total dependência da radiação, respectivamente (Goldberg \& Bernhofer, 2008).

Entretanto, estudos do balanço de energia combinados ao controle da evapotranspiração ainda não foram aplicados à Caatinga, em épocas de seca intensa. Esses estudos são importantes, uma vez que permitem uma análise mais realística e detalhada da interação do ecossistema com a atmosfera; possibilitam uma melhor compreensão das respostas do ecossistema às mudanças no regime de precipitação e da assimilação de carbono pelo sistema; e servem como parâmetros de entrada em modelos de simulação climática.

O objetivo deste trabalho foi determinar a variação sazonal dos componentes do balanço de energia e avaliar o controle biofísico da evapotranspiração, em área de Caatinga preservada, em condições de seca intensa.

\section{Material e Métodos}

$\mathrm{O}$ experimento foi realizado em área de Caatinga $\left(9^{\circ} 05^{\prime} \mathrm{S}, 40^{\circ} 19^{\prime} \mathrm{W}\right.$, a $350 \mathrm{~m}$ de altitude), no Município de Petrolina, PE. O clima da região, segundo Köppen, é do tipo BSwh', ou seja, semiárido com estação chuvosa compreendida entre janeiro e abril, precipitação média anual de $510 \mathrm{~mm}$ e temperaturas médias anuais da ordem de $26,2^{\circ} \mathrm{C}$. A área em estudo apresenta relevo plano e extensão total de aproximadamente 600 ha, composta por vegetação hiperxerófila de porte arbóreo-arbustivo, com altura média de 5,0 m.

Os aspectos micrometeorológicos foram caracterizados para o período de 1/1/2012 a $31 / 12 / 2012$, o que totalizou 366 dias, por meio do sistema "eddy covariance", instalado em uma torre de $16 \mathrm{~m}$ de altura. Buscou-se garantir uma bordadura de pelo menos $300 \mathrm{~m}$ em todas as direções em relação ao ponto de medição, especialmente na direção do vento predominante, para minimizar possíveis erros de medição. A área do "footprint" foi calculada com uso do modelo de Hsieh et al. (2000). Constatou-se que a contribuição da direção do vento predominante, durante a maior parte do período, ocorreu nas proximidades da torre, entre 120 e $200 \mathrm{~m}$, e que este foi proveniente do sudeste. Assim, os sensores foram orientados na direção do vento médio vertical, ao lado do ponto de medição, para minimizar a distorção do fluxo.

As medições do fluxo de calor sensível e de calor latente foram realizadas a $16,9 \mathrm{~m}$ acima da superfície do solo, tendo-se utilizado o sistema "eddy covariance" composto por anemômetro sônico tridimensional, modelo CSAT-3 (Campbell Scientific Inc., Logan, UT, EUA), para medir as flutuações médias da velocidade de vento ( $u, v$, e w) e da temperatura. Também foi utilizado analisador de gás por infravermelho de caminho aberto, modelo LI-7500 (Li-Cor Inc., Lincoln, NE, EUA), para medidas da concentração do 
vapor d'água; ambos os equipamentos operaram a uma frequência de $16 \mathrm{~Hz}$.

As medidas dos elementos meteorológicos foram realizadas com sensores ligados ao sistema de aquisição de dados CR1000 (Campbell Scientific Inc., Logan, UT, EUA), programado para realizar medidas a cada $60 \mathrm{~s}$ com médias em intervalos de $10 \mathrm{~min}$. Para a determinação dos componentes do balanço de radiação, foi utilizado saldo radiômetro, modelo CNR1 (Kipp \& Zonen, Delft, Holanda), instalado a 13,3 $\mathrm{m}$ acima da superfície do solo. A temperatura e a umidade relativa do ar foram medidas com sensor HMP45C (Campbell Scientific Inc., Logan, UT, EUA), a 15,7 $\mathrm{m}$ acima da superfície do solo, e a precipitação, com pluviômetro CS700-L (Campbell Scientific Inc., Logan, UT, EUA), a 16,3 m. Já a temperatura do solo foi obtida a 0,02 e $0,08 \mathrm{~m}$ de profundidade, com o sensor CS107 (Campbell Scientific Inc., Logan, UT, EUA), e a velocidade do vento, por meio do anemômetro sônico WindMaster Pro (Gill Instruments Limited, Lymington, Reino Unido), a 16,9 m de altura.

Os dados dos fluxos de calor sensível e latente foram utilizados no cálculo das médias de $30 \mathrm{~min}$. $\mathrm{O}$ processamento dos dados brutos incluiu: rotação das coordenadas, e correção da flutuação da densidade, da umidade e da temperatura do ar, de acordo com Mauder et al. (2008) e Zeri \& Sá (2011); estes procedimentos foram realizados por meio do programa Alteddy, versão 3.6 (Alterra, Wageningen, Holanda). Também foram feitos testes básicos para analisar as condições de estabilidade da atmosfera, tendo-se aplicado filtros na velocidade de fricção $\left(\mathrm{u}^{*}<0,5 \mathrm{~m} \mathrm{~s}^{-1}\right)$, para minimizar as condições de advecção e baixa turbulência (Foken et al., 2004). Após esse procedimento, constatou-se que cerca de $80 \%$ dos dados apresentaram consistência para utilização nas análises subsequentes.

Posteriormente, os dados faltantes foram preenchidos com uso da ferramenta online "eddy covariance gap-filling \& flux-partitioning tool" (Max Planck Institute for Biogeochemistry, Jena, Alemanha), como proposto por Falge et al. (2001), tendo-se considerado a covariância entre os fluxos e as variáveis meteorológicas (Reichstein et al., 2005). Dessa forma, os dados faltantes foram preenchidos por um valor médio em condições meteorológicas semelhantes, num intervalo de tempo de 7 dias.
A partição do saldo de radiação $\left(\mathrm{Rn}\right.$, em $\left.\mathrm{W} \mathrm{m}^{-2}\right)$, representada pelos fluxos de calor sensível e de calor latente, foi obtida com base nas seguintes equações:

$\mathrm{Rn}-\mathrm{H}-\mathrm{LE}-\mathrm{G}=0, \mathrm{H}=\rho_{\mathrm{a}} \mathrm{c}_{\mathrm{p}} \overline{\mathrm{w}^{\prime} \mathrm{T}^{\prime}}$ e $\mathrm{LE}=\mathrm{L} \overline{\mathrm{W}^{\prime} \mathrm{q}^{\prime}}$, em que $\mathrm{H}$ é o fluxo de calor sensível ( $\left.\mathrm{W} \mathrm{m}^{-2}\right)$; LE é o fluxo de calor latente $\left(\mathrm{W} \mathrm{m}^{-2}\right)$; $\mathrm{G}$ é o fluxo de calor no solo $\left(\mathrm{W} \mathrm{m}^{-2}\right)$; w' é a variação da velocidade vertical do vento; L é o calor latente de evaporação; $\rho_{\mathrm{a}}$ é densidade do ar $\left(\mathrm{g} \mathrm{m}^{-3}\right) ; \mathrm{c}_{\mathrm{p}}$ é o calor específico do $\operatorname{ar}\left(\mathrm{J} \mathrm{kg}^{-1} \mathrm{~K}^{-1}\right)$; T é a temperatura do ar $\left({ }^{\circ} \mathrm{K}\right)$; e q é a umidade especifica $\left(\mathrm{g} \mathrm{g}^{-1}\right)$.

Os dados do fluxo de calor latente foram utilizados no cálculo da evapotranspiração real (ETr). Além disso, foi determinada a evapotranspiração de referência (ETo) por meio do modelo de Penman-Monteith-FAO, tendo-se utilizado dados medidos na Estação Agrometeorológica Automática do Campo Experimental da Caatinga, localizada à cerca de 4,0 km da torre micrometeorológica.

Para melhorar a compreensão dos fatores que governam a evapotranspiração, foram calculados alguns parâmetros em nível da superfície: resistência da superfície e fator de desacoplamento (McNaughton \& Jarvis, 1983).

A resistência da superfície $\left(\mathrm{r}_{\mathrm{s}}, \mathrm{em} \mathrm{s} \mathrm{m}^{-1}\right)$ é indicativa da influência da superfície na perda de calor latente e foi obtida por meio da reorganização da equação de Penman-Monteith:

$$
r_{s}=\frac{\rho_{a} c_{p} V D P}{\gamma L E}-r_{a} \frac{1-\Delta H}{\gamma L E},
$$

em que $\rho_{\mathrm{a}}$ é a massa específica do ar $\left(\mathrm{kg} \mathrm{m}^{-3}\right)$; $\mathrm{c}_{\mathrm{p}}$ é o calor específico do ar úmido à pressão constante $\left(\mathrm{J} \mathrm{kg}^{-1}{ }^{\circ} \mathrm{C}^{-1}\right)$; VPD é o deficit de pressão de vapor $(\mathrm{kPa})$; LE é o calor latente de evaporação $\left(\mathrm{W} \mathrm{m}^{-2}\right)$; H é o fluxo de calor sensível $\left(\mathrm{W} \mathrm{m}^{-2}\right) ; \Delta$ é a declividade da curva de pressão de saturação $\left(\mathrm{kPa}^{\circ} \mathrm{C}^{-1}\right)$; e $\gamma$ é o coeficiente psicrométrico $\left(\mathrm{Pa}^{\circ} \mathrm{K}^{-1}\right)$.

O fator de desacoplamento $(\Omega)$ foi determinado pela metodologia proposta por McNaughton \& Jarvis (1983), com base na equação:

$$
\Omega=\left(1+\frac{\gamma}{\gamma+\Delta} \frac{r_{s}}{r_{a}}\right)^{-1},
$$

em que $\gamma$ é o coeficiente psicrométrico $\left(\mathrm{Pa}^{\circ} \mathrm{K}^{-1}\right) ; \Delta$ é a declividade da curva de pressão de saturação de vapor d'água $\left(\mathrm{k} \mathrm{Pa}^{\circ} \mathrm{C}^{-1}\right)$; e $\mathrm{r}_{\mathrm{s}}$ e $\mathrm{r}_{\mathrm{a}}$ representam as resistências da superfície e aerodinâmica $\left(\mathrm{s} \mathrm{m}^{-1}\right)$, respectivamente. Nesse caso, para obtenção da resistência aerodinâmica, 
foram consideradas as correções de estabilidade atmosférica para o fluxo de momentum $\left(\psi_{\mathrm{m}}\right)$ e de calor $\left(\psi_{\mathrm{h}}\right)$, sugeridas por Arya (2001). Assim, a resistência aerodinâmica $\left(\mathrm{r}_{\mathrm{a}}, \mathrm{em} \mathrm{s} \mathrm{m}^{-1}\right)$ foi obtida de acordo com a equação (Campbell \& Norman, 1998):

$$
\mathrm{r}_{\mathrm{a}}=\frac{\left[\ln \left(\frac{\mathrm{z}_{\mathrm{m}}-\mathrm{d}}{\mathrm{z}_{\mathrm{om}}}\right)-\psi_{\mathrm{m}}\right] \cdot\left[\ln \left(\frac{\mathrm{z}_{\mathrm{h}}-\mathrm{d}}{\mathrm{z}_{\mathrm{oh}}}\right)-\psi_{\mathrm{h}}\right]}{\mathrm{k}^{2} \cdot \mathrm{U}_{\mathrm{z}}},
$$

em que $\mathrm{z}_{\mathrm{m}}$ é a altura de medição da velocidade do vento $(\mathrm{m})$; d é o deslocamento do plano zero (m); $z_{\text {om }}$ é o comprimento da rugosidade para momentum (m); $z_{\mathrm{h}}$ é a altura de medição de umidade (m); $\mathrm{z}_{\mathrm{oh}}$ é o comprimento de rugosidade da transferência de calor e vapor $(\mathrm{m})$; k é a constante de von Karman $(\mathrm{k}=0,41)$; e $\mathrm{U}_{\mathrm{z}}$ é a velocidade do vento na altura $\mathrm{z}(\mathrm{m})$.

O índice de cobertura vegetal (ICV) foi determinado em intervalos médios de 15 dias, ao longo de 2012. Foi utilizado o ceptômetro AccuPar (Decagon Devices Inc., Pullman, WA, EUA), que estima o ICV em função da interceptação luminosa pelo dossel. Para garantir a precisão das leituras, as medidas foram realizadas em horários sempre próximos ao meio dia local, em três direções: nordeste, noroeste e sudeste, tendo-se efetuado 12 leituras em cada direção, o que totalizou 36 repetições por campanha de medição.

\section{Resultados e Discussão}

Em 2012, foi registrada uma lâmina de precipitação pluvial anual acumulada de $92 \mathrm{~mm}$, com 90\% deste total ocorridos entre janeiro e maio, cujo valor é muito inferior ao da normal climatológica da região, de $510 \mathrm{~mm}$ anuais (Médias..., 2014). Essa redução significativa de precipitação tem sido atribuída, principalmente, à variação da temperatura na superfície do Oceano Atlântico, ocasionada pelo Dipolo do Atlântico, que interferiu no deslocamento da Zona de Convergência Intertropical e, consequentemente, no regime pluvial da região Nordeste (Centro..., 2014; Gutiérrez et al., 2014). Como resultado da redução da precipitação, foram observadas alterações em todos os elementos meteorológicos durante 2012. O ano iniciou com valores elevados de radiação solar global (Qg), que atingiu, em média, $23 \mathrm{MJ} \mathrm{m}^{-2}$ por dia. Com a mudança nas estações do ano, houve redução da Qg durante o inverno, seguindo uma tendência de aumento durante a primavera e o verão (Souza et al., 2015). Nesse ano especificamente, os valores de radiação foram superiores à normal da região, de $20 \mathrm{MJ} \mathrm{m}^{-2}$ por dia. Esse comportamento da radiação exerceu influência direta na temperatura do solo, que atingiu valores médios diários que oscilaram entre $34^{\circ} \mathrm{C}$, em janeiro, e $38^{\circ} \mathrm{C}$, em dezembro, com mínimos próximos de $26^{\circ} \mathrm{C}$, em fevereiro. A temperatura e a umidade relativa do ar apresentaram pequena variação ao longo do ano, com valores médios diários de $27^{\circ} \mathrm{C}\left( \pm 1,5^{\circ} \mathrm{C}\right)$ e $49 \%( \pm 4,2 \%)$, respectivamente. Isso resultou em um deficit de pressão de vapor que atingiu valores mínimos de $1,42 \mathrm{kPa}$, em alguns dias de fevereiro, e máximos de $2,39 \mathrm{kPa}$, em dezembro, com valor médio anual de $1,95 \mathrm{kPa}$.

O padrão sazonal do saldo de radiação em 2012 seguiu aproximadamente a variação da $\mathrm{Qg}$, tendo apresentado valores totais diários máximos, médios e mínimos em torno de 18,11 e $6 \mathrm{MJ} \mathrm{m}^{-2}$ por dia, respectivamente. Em média, durante 2012, observouse que a maior parte da energia disponível foi destinada ao fluxo de calor sensível (Figura 1). Os valores diários do fluxo de calor no solo foram quase sempre positivos, com média de $0,16 \pm 0,28 \mathrm{MJ} \mathrm{m}^{-2}$ por dia. No entanto, esses valores representaram apenas $3 \%$ da energia disponível no sistema, com pouca variação ao longo do ano. Nos dias em que houve chuva, o fluxo de calor no solo atingiu valores mínimos de $-1,28 \mathrm{MJ} \mathrm{m}^{-2}$ por dia, o que indica perda de energia da superfície do solo para a atmosfera.

A variação temporal do fluxo de calor sensível (H), contudo, foi correspondente ao padrão do saldo de radiação $(\mathrm{Rn})$. Neste caso, a partição $\mathrm{H} / \mathrm{Rn}$ foi, em média, $77 \%$ durante o ano, tendo oscilado entre $63 \%$, nos meses de maior disponibilidade de água, e $89 \%$ nos meses mais secos do ano (Figura 2).

A maior utilização da energia disponível para o fluxo de calor sensível, em ecossistemas naturais, tem sido um comportamento bastante evidenciado em regiões áridas e semiáridas, em decorrência da redução na disponibilidade hídrica ocasionada pelos baixos volumes pluviais nesses locais (Santos et al., 2012; Teixeira et al., 2008). Teixeira et al. (2008), em estudo realizado em área de Caatinga em Petrolina, PE, em 2004 e 2005, evidenciaram uma partição da energia para o fluxo de calor sensível em torno de 49 e $63 \%$, respectivamente. No presente trabalho, o aumento deste componente pode ser explicado pelo aumento 


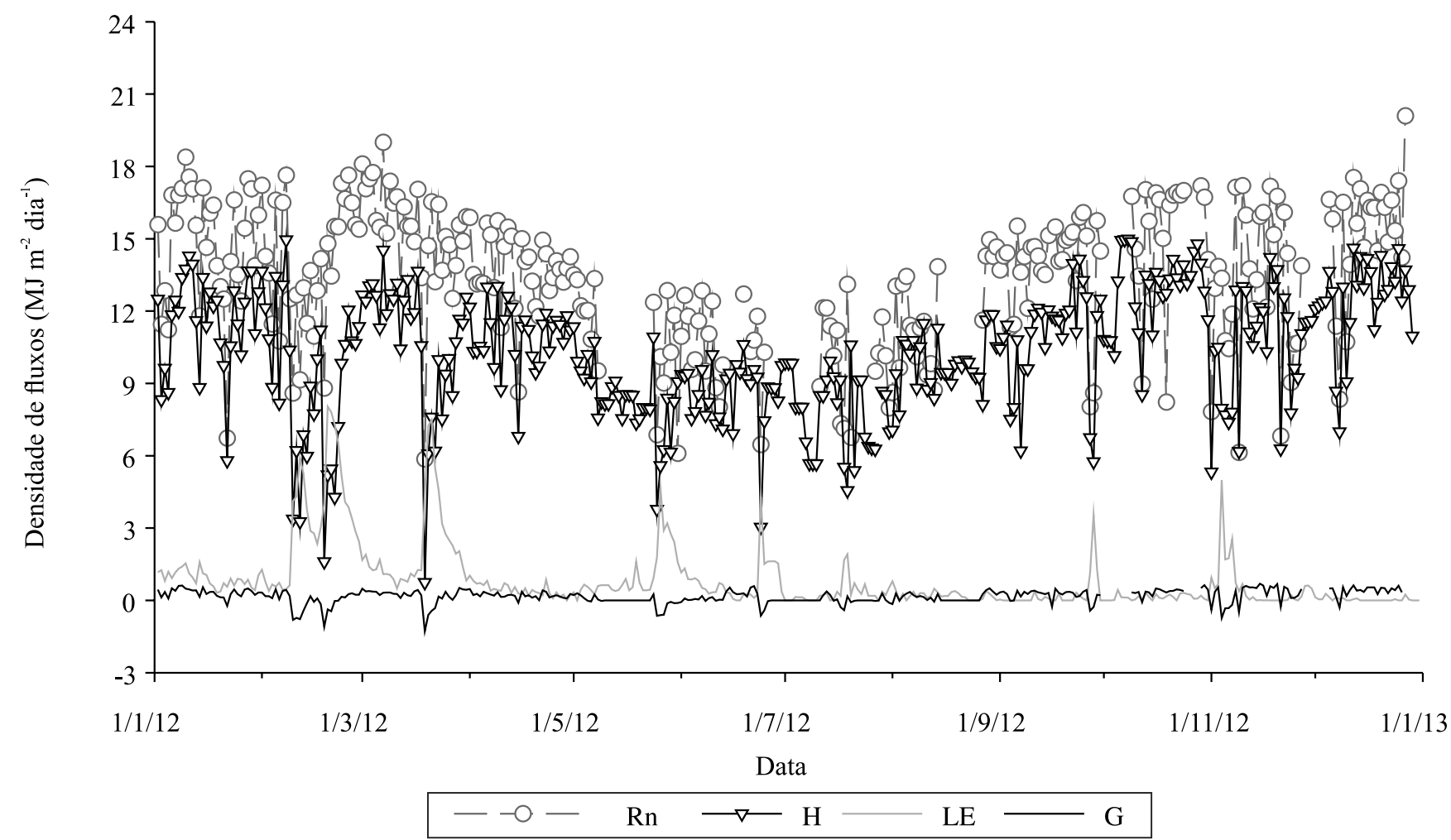

Figura 1. Densidade de fluxos de calor latente (LE), de calor sensível (H) e de calor no solo (G) durante 2012, em área de Caatinga preservada. Rn, saldo de radiação.

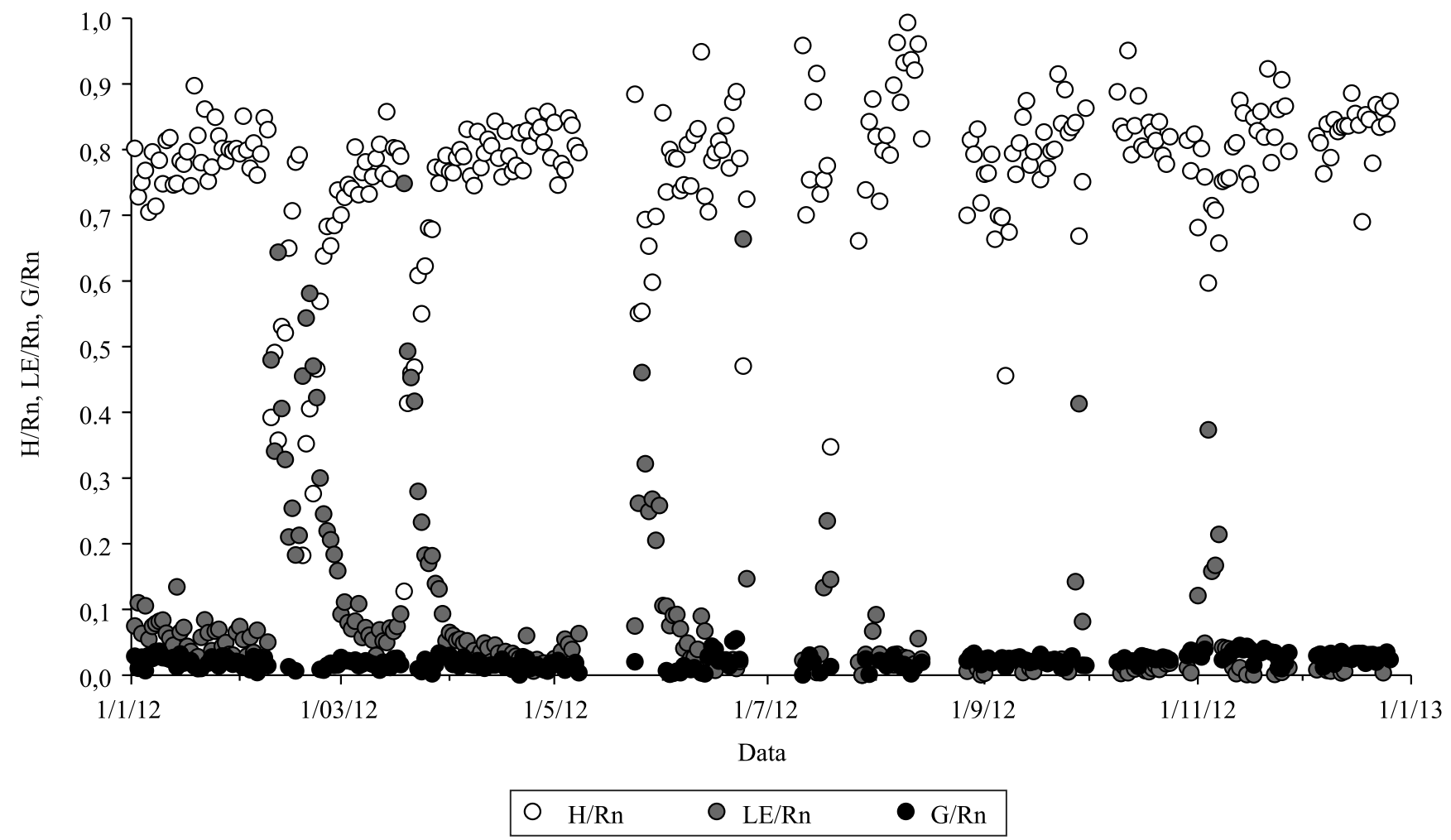

Figura 2. Partição do saldo de radiação (Rn) nos fluxos de calor latente (LE), de calor sensível (H) e de calor no solo (G), durante 2012, em área de Caatinga preservada. 
da deficiência hídrica no sistema solo-atmosfera, resultante dos baixos volumes pluviais registrados em 2012. Nessas condições, a redução da disponibilidade hídrica culminou no aumento da fração da energia destinada ao aquecimento do ambiente.

O fluxo de calor latente (LE) apresentou baixos valores no início do ano, decorrentes da baixa disponibilidade de água no ecossistema, tendo atingido valores médios diários que oscilaram de $3,3 \mathrm{MJ} \mathrm{m}^{-2}$ por dia $(\mathrm{LE} / \mathrm{Rn}=25 \%)$, em fevereiro, a $0,08 \mathrm{MJ} \mathrm{m}^{-2}$ por dia em dezembro (Figura 1). Apenas em dias em que ocorreram eventos de precipitação, os valores diários de fluxo de calor latente foram mais elevados, tendo atingido máximos em torno de $8 \mathrm{MJ} \mathrm{m}^{-2}$ por dia $(\mathrm{LE} / \mathrm{Rn}=60 \%)$. Posteriormente, a redução na disponibilidade de água no solo provocou novamente a diminuição do fluxo de calor latente, que atingiu valores médios diários em torno de $0,03 \mathrm{MJ} \mathrm{m}^{-2}$ por dia, o que representou cerca de apenas $3 \%$ da energia disponível (LE/Rn), durante 8 meses do ano, e, em média, 7\% durante o ano inteiro (Figura 2). Esses resultados são bastante inferiores aos obtidos por Teixeira et al. (2008), que, em estudo realizado em 2004 e 2005, encontraram valores médios diários ( 24 horas) iguais a 4,62 e 2,67 $\mathrm{MJ} \mathrm{m}^{-2}$ por dia, respectivamente. Essas diferenças entre os valores de fluxo de calor latente obtidos no presente trabalho e os relatados na literatura são esperadas, uma vez que, em 2012, houve restrição hídrica intensa no sistema solo-atmosfera. Nessas condições, os valores de fluxo de calor latente foram severamente reduzidos, e essa informação é bastante útil para o entendimento de possíveis mudanças no sistema climático local em virtude de alterações na disponibilidade hídrica local.

Observou-se boa correlação entre os valores de fluxos medidos pelo sistema "eddy covariance" e os medidos pelos sensores micrometeorológicos (Rn - G), com $\mathrm{R}^{2}=0,91$ (Figura 3). O valor médio da razão entre $(\mathrm{LE}+\mathrm{H})$ e $(\mathrm{Rn}-\mathrm{G})$ foi de $75 \%$, com raiz quadrada do erro quadrático médio (RMSE) de $5,6 \mathrm{~W} \mathrm{~m}^{-2}$, que correspondeu a $25 \%$ da energia disponível $(\mathrm{Rn}-\mathrm{G})$. Esses resultados são satisfatórios diante do grande número de dados analisados. Frequentemente, erros no fechamento entre 10 e $30 \%$ têm sido relatados na literatura (Wilson et al., 2002). Estes mesmos autores analisaram o fechamento do balanço de energia pelo método "eddy covariance", em 22 localidades, com períodos de observações de 1 a 4 anos, e observaram desigualdades entre $(\mathrm{Rn}-\mathrm{G})$ e $(\mathrm{H}+\mathrm{LE})$, que variaram de 15 a 30\%, com média de 20\%. Teixeira et al. (2010), ao analisar o fechamento do balanço de energia em escala diária, em área de Caatinga, encontraram valor de RMSE igual a 1,7 $\mathrm{MJ} \mathrm{m}^{-2}$ por dia, equivalente ao erro de $10 \%$, enquanto Oliveira et al. (2006) obtiveram erro igual a $15 \%$, como uso de dados horários.

$\mathrm{O}$ não fechamento do balanço de energia em regiões semiáridas e áridas tem sido atribuído a vários fatores, como a não inclusão de outros sumidouros de energia (Wilson et al., 2002; Li et al., 2006) e o gradiente de temperatura nas camadas superiores do solo, que promovem, entre outros fatores, o armazenamento de calor nas camadas acima do sensor (Wilson et al., 2002; Veenendaal et al., 2004; Oliveira et al., 2006).

$\mathrm{Na}$ análise sazonal da ETr da Caatinga, foram observados valores bastante inferiores aos da ETo, que representaram, em média, cerca de $7 \%$ da ETo, em decorrência da baixa disponibilidade hídrica observada na região (Figura 4). Em alguns dias de fevereiro, esse comportamento se inverteu, e verificou-se que a ETo apresentou valores inferiores aos da ETr. Nesse caso, os valores da ETo mais reduzidos podem ser atribuídos à ocorrência de chuvas e, consequentemente, à demanda atmosférica; já a ETr apresentou valores superiores em função da disponibilidade de água no sistema.

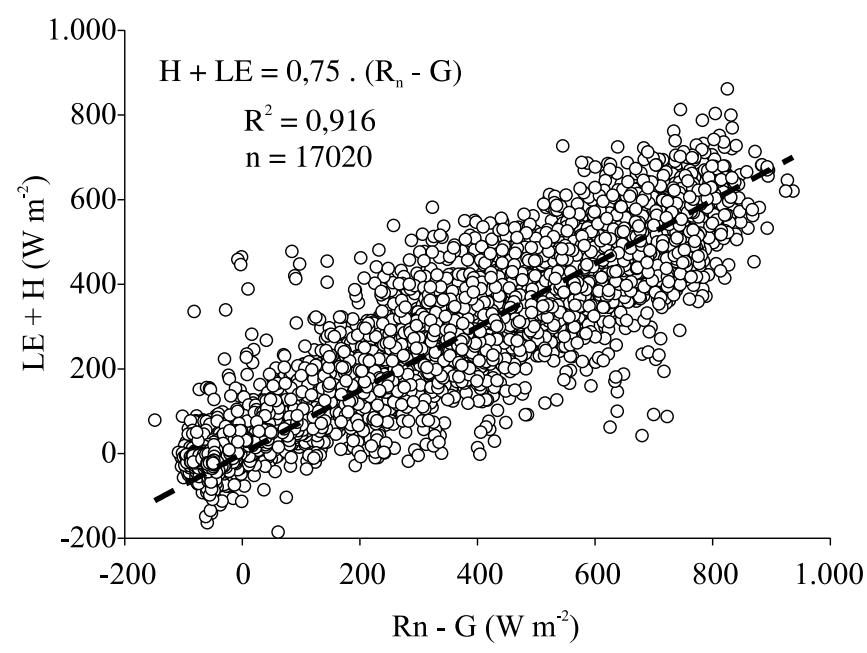

Figura 3. Relação entre os dados horários da energia disponível no sistema $(\mathrm{Rn}-\mathrm{G})$ obtida por meio de sensores micrometeorológicos, e soma dos fluxos de calor sensível e de calor latente $(\mathrm{LE}+\mathrm{H})$ pela técnica de eddy covariance ( $\mathrm{n}=$ número de dados), em área de Caatinga preservada. $\mathrm{Rn}$, saldo de radiação; e $\mathrm{G}$, fluxo de calor no solo. 
No início do ano, mesmo com a presença de folhas na vegetação ( $\mathrm{ICV}=2,64)$, foram observados valores baixos de ETr, com média diária igual a $0,5 \mathrm{~mm}$ por dia. Do final de fevereiro até meados de março, ocorreram alguns eventos de chuva e houve aumento da disponibilidade hídrica no ecossistema, o que possibilita incremento da ETr, que atingiu valores máximos diários de 3,0 $\mathrm{mm}$ por dia. Nos dias seguintes, a demanda atmosférica substancialmente elevada (deficit de pressão de vapor $=2,2 \mathrm{kPa}$ ) ocasionou redução na disponibilidade hídrica no sistema $\mathrm{e}$ diminuição nos valores da $\mathrm{ETr}$, os quais permaneceram aproximadamente nulos. Portanto, apesar do longo período de completa restrição hídrica observado, de cerca de 4 meses, houve grande capacidade das espécies em responder às variações no ambiente, com recuperação rápida da biomassa foliar e resposta da ETr em novembro (2,0 $\mathrm{mm}$ por dia).

A Caatinga apresenta resistência à perda de água para a atmosfera, representada pela $r_{s}$, que atingiu valores máximos diários de até $9.000 \mathrm{~s} \mathrm{~m}^{-1}$, com média anual de $2.377 \mathrm{~s} \mathrm{~m}^{-1}$ (Figura 5). Teixeira et al. (2008), ao avaliar a Caatinga em um ano chuvoso, com precipitação anual de $720 \mathrm{~mm}$, obtiveram valores de $\mathrm{r}_{\mathrm{s}}$ iguais a $1.542 \mathrm{~s} \mathrm{~m}^{-1}$, enquanto em 2005, quando a precipitação foi próxima da normal climatológica da região $(\mathrm{P}=525 \mathrm{~mm})$, os valores de $r_{s}$ foram de $2.332 \mathrm{~s} \mathrm{~m}^{-1}$, com valores máximos próximos de $7.200 \mathrm{~s} \mathrm{~m}^{-1}$, a partir de agosto. Os altos valores registrados no presente trabalho mostram a capacidade da vegetação em se ajustar às variações na atmosfera, tendo apresentado valores de ETr sempre em torno de $0,5 \mathrm{~mm}$ por dia.

A sensibilidade da vegetação à atmosfera pode ser constatada pelos baixos valores do $\Omega$, com média diária em torno de 0,14 , durante janeiro, quando a Caatinga apresentava ICV de 2,64 $\pm 0,08$, tendo atingido valores mínimos e máximos diários de 0,04 a 0,38 , respectivamente. Esses valores oscilaram nesse intervalo, em função das modificações no

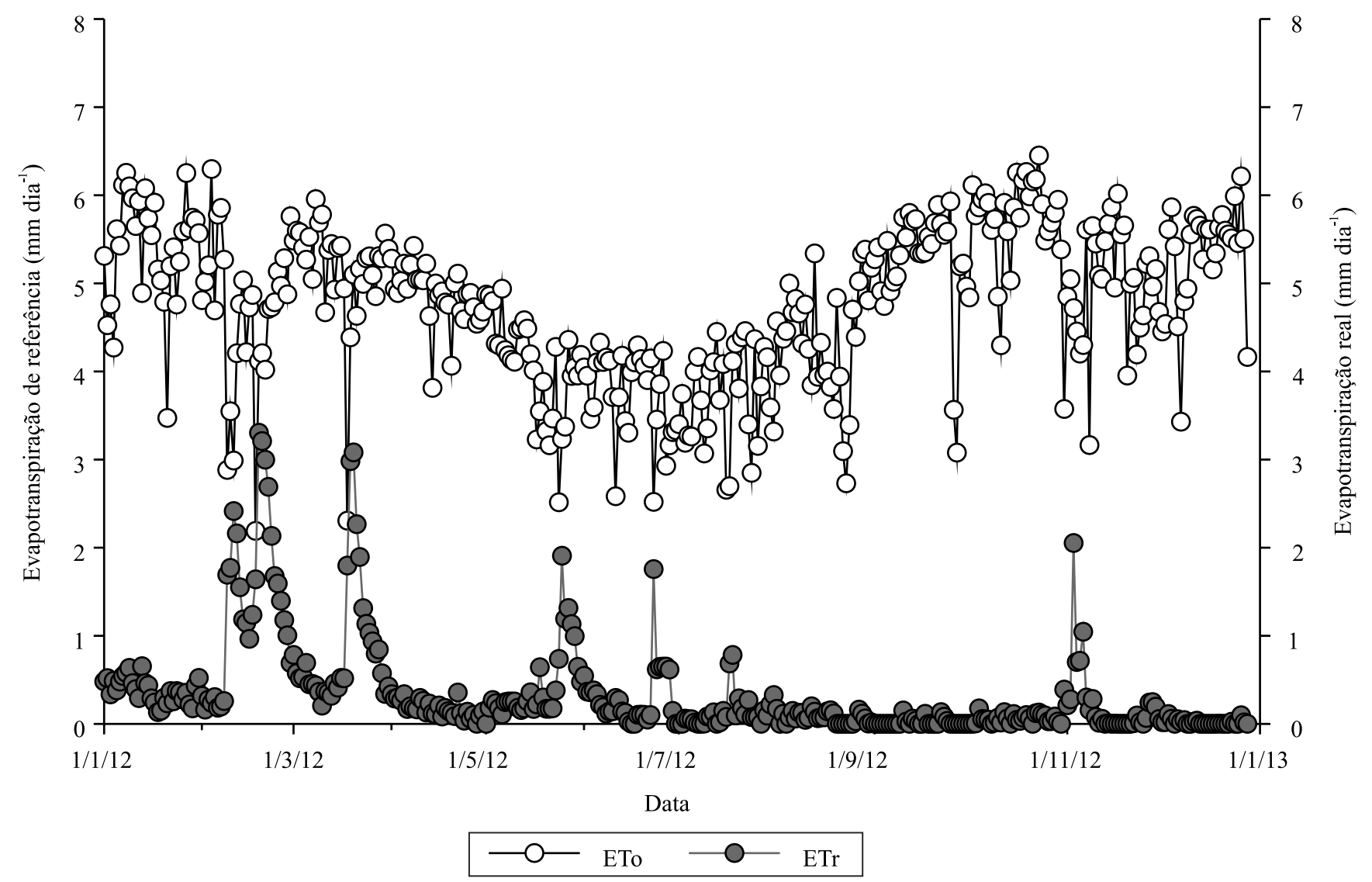

Figura 4. Variação sazonal da evapotranspiração de referência (ETo) e da evapotranspiração real (ETr) de área de Caatinga preservada durante 2012 . 
deficit de pressão de vapor e na velocidade do vento. $O$ valor do $\Omega$ foi maior $(0,38)$ quando a velocidade do vento e o deficit de pressão de vapor foram menores. A intensificação dessas duas variáveis induz o maior acoplamento entre a superfície e a atmosfera, $\operatorname{com} \Omega$ tendendo a 0 (Figura 5), como constatado por Silva et al. (2012), em trabalho desenvolvido com cana-de-açúcar (Saccharum officinarum L.) no Semiárido brasileiro.

No final de fevereiro e março, por ocasião de eventos de precipitação, um incremento da ETr, de $3,0 \mathrm{~mm}$ por dia, resultou no aumento do $\Omega$. Neste caso, a presença de água no sistema ocasionou resposta rápida da vegetação, que se mostrou desacoplada da atmosfera $(\Omega=1)$, tendo apresentado $r_{s}$ praticamente nula e $\mathrm{r}_{\mathrm{a}}$ alta, em torno de $50 \mathrm{~s} \mathrm{~m}^{-1}$. Esses resultados são indicativos de que a energia disponível tem maior importância do que o controle estomático no processo de evapotranspiração, quando há disponibilidade de água na Caatinga. O comportamento da ETr, nesse caso, foi governado especialmente pela radiação solar e pela $r_{a}$, com valores de $\Omega=1$. A resistência imposta pela superfície atingiu, nesses dias, os menores valores anuais, em torno de $5 \mathrm{~s} \mathrm{~m}^{-1}$, que indicaram o maior desacoplamento da atmosfera. Resultados semelhantes foram obtidos por Silva et al. (2012), que, ao analisar o fator de desacoplamento em um agroecossistema de cana-de-açúcar, observaram que o final do período chuvoso favoreceu o incremento dos valores do fator de desacoplamento, possivelmente em razão da presença de água, que culminou em menor controle da vegetação sobre a transferência de vapor d'água para a atmosfera.

Em dias secos, que compreenderam a maior parte do período avaliado no presente trabalho, ou seja, 349 dias, verificou-se que a ETr foi governada principalmente pelo deficit de pressão de vapor (DPV médio anual $=1,95 \mathrm{kPa}$ ) e pela $\mathrm{r}_{\mathrm{s}}$, com valores de $\Omega$ próximos de zero (Figura 5). O comportamento do $\Omega$ mostra a existência de um forte acoplamento entre a superfície e a atmosfera.

Um bom acoplamento entre a superfície e a vegetação normalmente tem sido observado quando esta apresenta porte oscilando de médio a alto, com altos valores de $r_{s}$, copa aberta e folhas pequenas, onde os valores de $\Omega$ oscilam entre 0 e 0,2 (Martin et al., 2001). Semelhante ao observado para a Caatinga. Nesses casos, a ocorrência de valores praticamente nulos pode ser atribuída, dentre outros fatores, à alta capacidade da vegetação de se ajustar às condições ambientais estressantes, especialmente ao déficit hídrico.

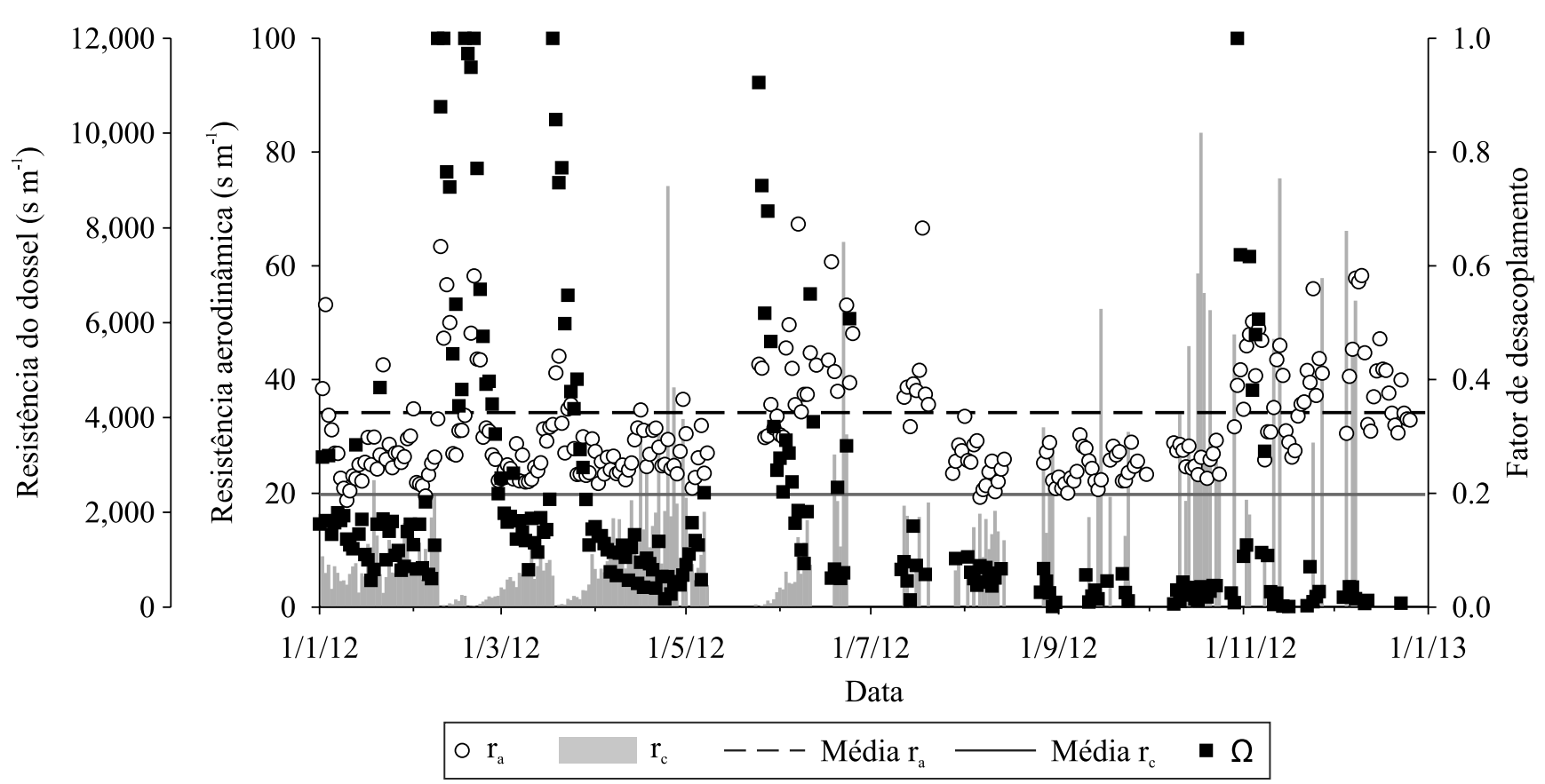

Figura 5. Comportamento das resistências aerodinâmica $\left(r_{a}\right)$ e da superfície $\left(r_{s}\right)$, e do fator de desacoplamento $(\Omega)$ de área de Caatinga preservada durante 2012. 


\section{Conclusões}

1. A partição dos componentes do balanço de energia destinada ao fluxo de calor sensível é maximizada pela ocorrência de seca prolongada.

2. A evapotranspiração em condições de seca intensa é especialmente dependente do deficit de pressão de vapor e da resistência da superfície.

3. Após demanda atmosférica elevada em área de Caatinga, a superfície apresenta grande capacidade de resposta ao ambiente.

\section{Agradecimentos}

Ao Conselho Nacional de Desenvolvimento Científico e Tecnológico $(\mathrm{CNPq}, \quad$ Processo no 483223/2011-5), pelo apoio financeiro.

\section{Referências}

ARYA, S.P. Introduction to micrometeorology. $2^{\text {nd }}$ ed. San Diego: Academic Press, 2001. 420p. (International geophysics series, 79).

CAMPBELL, G.S.; NORMAN, J.M. An introduction to environmental biophysics. New York: Springer, 1998. DOI: 10.1007/978-1-4612-1626-1.

CENTRO de Previsão de Tempo e Estudos Climáticos. Disponível em: $<$ http://www.cptec.inpe.br>. Acesso em: 3 set. 2014.

CHEN, S.; CHEN, J.; LIN, G.; ZHANG, W.; MIAO, H.; WEI, L.; HUANG, J.; HAN, X. Energy balance and partition in Inner Mongolia steppe ecosystems with different land use types. Agricultural and Forest Meteorology, v.149, p.1800-1809, 2009. DOI: 10.1016/j.agrformet.2009.06.009.

CUNHA, A.P.M. do A.; ALVALÁ, R.C. dos S.; OLIVEIRA, G.S. de. Impactos das mudanças de cobertura vegetal nos processos de superfície na região semiárida do Brasil. Revista Brasileira de Meteorologia, v.28, p.139-152, 2013. DOI: 10.1590/ S0102-77862013000200003.

FALGE, E.; BALDOCCHI, D.; OLSON, R.; ANTHONI, P.; AUBINET, M.; BERNHOFER, C.; BURBA, G.; CEULEMANS, R.; CLEMENT, R.; DOLMAN, H.; GRANIER, A.; GROSS, P.; GRÜNWALD, T.; HOLLINGER, D.; JENSEN, N.-O.; KATUL, G.; KERONEN, P.; KOWALSKI, A.; LAI, C.T.; LAW, B.E.; MEYERS, T.; MONCRIEFF, J.; MOORS, E.; MUNGER, J.W.; PILEGAARD, K.; RANNIK, Ü.; REBMANN, C.; SUYKER, A.; TENHUNEN, J.; TU, K.; VERMA, S.; VESALA, T.; WILSON, K.; WOFSKY, S. Gap filling strategies for defensible annual sums of net ecosystem exchange. Agricultural and Forest Meteorology, v.107, p.43-69, 2001. DOI: 10.1016/S0168-1923(00)00225-2.

FOKEN, T.; GÖCKEDE, M.; MAUDER, M.; MAHRT, L.; AMIRO, B.; MUNGER, W. Post-field data quality control. In: LEE, X.; MASSMAN, W.; LAW, B. (Ed.). Handbook of micrometeorology: a guide for surface flux measurements. Dordrecht: Kluwer Academic, 2004. p.81-108.
GOLDBERG, V.; BERNHOFER, C. Testing different decoupling coefficients with measurements and models of contrasting canopies and soil water conditions. Annales Geophysicae, v.26, p.1977-1992, 2008. DOI: 10.5194/angeo-26-1977-2008.

GUTIÉRREZ, A.P.A.; ENGLE, N.L.; NYS, E.D.; MOLEJÓN, C.; MARTINS, E.S. Drought preparedness in Brazil. Weather and Climate Extremes, v.3, p.95-106, 2014. DOI: 10.1016/j. wace.2013.12.001.

HSIEH, C.-I.; KATUL, G.; CHI, T.-W. An approximate analytical model for footprint estimation of scalar fluxes in thermally stratified atmospheric flows. Advances in Water Resources, v.23, p.765-772, 2000. DOI: 10.1016/S0309-1708(99)00042-1.

HU, Z.M.; YU, G.R.; ZHOU, Y.L.; SUN, X.M.; LI, Y.N.; SHI, P.L.; WANG, Y.F.; SONG, X.; ZHENG, Z.M.; ZHANG, L.; LI, S.G. Partitioning of evapotranspiration and its controls in four grassland ecosystems: application of a two-source model. Agricultural and Forest Meteorology, v.149, p.1410-1420, 2009. DOI: 10.1016/j. agrformet.2009.03.014.

HUIZHI, L.; JIANWU, F. Seasonal and interannual variations of evapotranspiration and energy exchange over different land surfaces in a semiarid area of China. Journal of Applied Meteorology and Climatology, v.51, p.1875-1888, 2012. DOI: 10.1175/JAMC-D-11-0229.1.

KRISHNAN, P.; MEYERS, T.P.; SCOTT, R.L.; KENNEDY, L.; HEUER, M. Energy exchange and evapotranspiration over two temperate semi-arid grasslands in North America. Agricultural and Forest Meteorology, v.152, p.31-44, 2012. DOI: 10.1016/j. agrformet.2011.09.017.

LI, S.-G.; EUGSTER, W.; ASANUMA, J.; KOTANI, A.; DAVAA, G.; OYUNBAATAR, D.; SUGITA, M. Energy partitioning and its biophysical controls above a grazing steppe in central Mongolia. Agricultural and Forest Meteorology, v.137, p.89-106, 2006. DOI: 10.1016/j.agrformet.2006.03.010.

MARIN, F.R.; ANGELOCCI, L.R. Irrigation requirements and transpiration coupling to the atmosphere of a citrus orchard in Southern Brazil. Agricultural Water Management, v.98, p.1091-1096, 2011. DOI: 10.1016/j.agwat.2011.02.002.

MARTIN, T.A.; BROWN, K.J.; KUCERA, J.; MEINZER, F.C.; SPRUGEL, D.G.; HINCKLEY, T.M. Control transpiration in a 220-year-old Abies amabilis forest. Forest Ecology and Management, v.152, p.211-224, 2001. DOI: 10.1016/ S0378-1127(00)00604-6.

MAUDER, M.; FOKEN, T.; CLEMENT, R.; ELBERS, J.A.; EUGSTER, W.; GRÜNWALD, T.; HEUSINKVELD, B.; KOLLE, O. Quality control of CarboEurope flux data - Part 2: inter-comparison of eddy-covariance software. Biogeosciences, v.5, p.451-462, 2008. DOI: 10.5194/bg-5-451-2008.

MCNAUGHTON, K.G.; JARVIS, P.G. Predicting effects of vegetation changes on transpiration and evaporation. In: KOZLOWSKI, T.T. (Ed.). Additional woody crop plants. New York: Academic Press, 1983. p.1-47. (Water deficits and plant growth, 7). DOI: 10.1016/b978-0-12-424157-2.50007-0.

MÉDIAS anuais da estação agrometeorológica de Bebedouro, Petrolina-PE. Período 1975-2014. [Ano 2013]. Petrolina: Embrapa Semiárido, [2014]. Disponível em: <http://www.cpatsa.embrapa. 
br:8080/servicos/dadosmet/ceb-anual.html>. Acesso em: 17 ago. 2014.

MOURA, M.S.B.; GALVÍNCIO, J.D.; BRITO, L.T. de L.; SOUZA, L.S.B. de; SÁ, I.I.S.; SILVA, T.G.F. da. Clima e água de chuva no Semi-Árido. In: BRITO, L.T. de L.; MOURA, M.S.B. de; GAMA, G.F.B. (Ed.). Potencialidades da água de chuva no Semi-Árido brasileiro. Petrolina: Embrapa Semi-Árido, 2007. p.37-59.

OLIVEIRA, M.B.L. de; SANTOS, A.J.B.; MANZI, A.O.; ALVALÁ, R.C. dos S.; CORREIA, M. de F.; MOURA, M.S.B. de. Trocas de energia e fluxo de carbono entre a vegetação de caatinga e atmosfera no Nordeste brasileiro. Revista Brasileira de Meteorologia, v.21, p.378-386, 2006.

PAIVA, S.C. de; CAVALCANTI, E.P. Fluxos de calor latente e sensível à superfície: estudo observacional e de simulação com o BRAMS no início do período de secas. Revista Brasileira de Geografia Física, v.4, p.677-691, 2011.

REICHSTEIN, M.; FALGE, E.; BALDOCCHI, D.; PAPALE, D.; AUBINET, M.; BERBIGIER, P.; BERNHOFER, C.; BUCHMANN, N.; GILMANOV, T.; GRANIER, A.; GRÜNEALD, T.; HAVRÁNKOVÁ, K.; ILVESNIEMI, H.; JANOUS, D.; KNOHL, A.; LAURILA, T.; LOHILA, A.; LOUSTAU, D.; MATTEUCI, G.; MEYERS, T.; MIGLIETTA, F.; OURCIVAL, J.M.; PUMPANEN, J.; RAMBAL, S.; ROTENBERG, E.; SANZ, M.; TENHUNEN, J.; SEUFERT, G.; VACCARI, F.; VESALA, T.; YAKIR, D.; VALENTINI, R. On the separation of net ecosystem exchange into assimilation and ecosystem respiration: review and improved algorithm. Global Change Biology, v.11, p.1424-1439, 2005. DOI: 10.1111/j.1365-2486.2005.001002.x.

SANTOS, S. de A.; CORREIA, M. de F.; ARAGÃO, M.R. da S.; SILVA, P.K. de O. Aspectos da variabilidade sazonal da radiação, fluxos de energia e $\mathrm{CO}_{2}$ em área de caatinga. Revista Brasileira de Geografia Física, v.4, p.761-773, 2012.

SILVA, T.G.F. da; ZOLNIER, S.; MOURA, M.S.B. de; CARMO, J.F.A. do; RIBEIRO, A. Fator de desacoplamento em um canavial irrigado no submédio do Vale do São Francisco. Revista Brasileira de Engenharia Agrícola e Ambiental, v.16, p.849-857, 2012. DOI: $10.1590 / \mathrm{S} 1415-43662012000800006$.
SOUZA, L.S.B. de; MOURA, M.S.B. de; SEDIYAMA, G.C.; SILVA, T.G.F. da. Balanço de radiação em ecossistema de Caatinga preservada durante um ano de seca no semiárido Pernambucano. Revista Brasileira de Geografia Física. v.8, p.41-55, 2015.

TEIXEIRA, A.H. de C. Determining regional actual evapotranspiration of irrigated crops and natural vegetation in the São Francisco River Basin (Brazil) using remote sensing and Penman-Monteith equation. Remote Sensing, v.2, p.1287-1319, 2010. DOI: $10.3390 / \mathrm{rs} 0251287$.

TEIXEIRA, A.H. de C.; BASTIAANSSEN, W.G.M.; AHMAD, M.D.; MOURA, M.S.B.; BOS, M.G. Analysis of energy fluxes and vegetation-atmosphere parameters in irrigated and natural ecosystems of semi-arid Brazil. Journal of Hydrology, v.362, p.110-127, 2008. DOI: 10.1016/j.jhydrol.2008.08.011.

VEENENDAAL, E.M.; KOLLE, O.; LLOYD, J. Seasonal variation in energy fluxes and carbon dioxide exchange for a broad-leaved semi-arid savanna (Mopane woodland) in Southern Africa. Global Change Biology, v.10, p.318-328, 2004. DOI: 10.1111/j.1365-248 6.2003.00699.x.

WILSON, K.; GOLDSTEIN, A.; FALGE, E.; AUBINET, M.; BALDOCCHI, D.; BERBIGIER, P.; BERNHOFER, C.; CEULEMANS, R.; DOLMAN, H.; FIELD, C.; GRELLE, A.; IBROM, A.; LAW, B.E.; KOWALSKI, A.; MEYERS, T.; MONCRIEFF, J.; MONSON, R.; OECHEL, W.; TENHUNEN, J.; VALENTINI, R.; VERMA, S. Energy balance closure at FLUXNET sites. Agricultural and Forest Meteorology, v.113, p.223-243, 2002. DOI: 10.1016/S0168-1923(02)00109-0.

YANG, F.; ZHOU, G.; HUNT, J.E.; ZHANG, F. Biophysical regulation of net ecosystem carbon dioxide exchange over a temperate desert steppe in Inner Mongolia, China. Agriculture Ecosystems and Environment, v.142, p.318-328, 2011. DOI: 10.1016/j.agee.2011.05.032.

ZERI, M.; SÁ, L.D. de A. Scale dependence of coherent structures' contribution to the daytime buoyancy heat flux over the Pantanal wetland, Brazil. Atmospheric Science Letters, v.12, p.200-206, 2011. DOI: 10.1002/asl.311.

Recebido em 26 de janeiro de 2015 e aprovado em 13 de julho de 2015

Pesq. agropec. bras., Brasília, v.50, n.8, p.627-636, ago. 2015

DOI: $10.1590 / \mathrm{S} 0100-204 X 2015000800001$ 